\title{
Making the links among environmental protection, process safety, and industry 4.0
}

\author{
Jose Alcides Gobbo Junior*, Christianne M. Busso, Simone Cristina O. Gobbo, \\ Henrique Carreão
}

UNESP - São Paulo State University, Production Engineering, 17033-360, Bauru, SP, Brazil

\section{A R T I C L E I N F O}

\section{Article history:}

Received 16 December 2017

Received in revised form 14 May 2018

Accepted 16 May 2018

Available online 21 May 2018

\section{Keywords:}

Industry 4.0

Environmental protection

Process safety

Bibliometric networks

\begin{abstract}
A B S T R A C T
Industry 4.0 is an emerging concept in production systems and is described as a concept that encompasses technologies such as the Internet of Things, big data, cyber-physical systems, and smart objects. Industry 4.0 will present new challenges and opportunities for process safety and environmental protection (PSEP) researchers and managers. There is a gap in the literature in identifying the main concepts related to industry 4.0 and PSEP, as well as the potential integration between these subjects. In line with this gap, this paper focuses on identifying and systemizing information regarding the integration of PSEP and industry 4.0 concepts and technologies. While identifying relationships, we also address non-existent intersections between keyword co-occurrence networks in PSEP and industry 4.0. The main outputs of this research are: (a) identification and discussion of potential connections between PSEP with industry 4.0 concepts; and (b) an in-depth discussion of potential benefits in integrating industry 4.0 concepts and technologies into PSEP fields. It can be concluded that there is much more collaborative research between environmental protection and industry 4.0 than between process safety and industry 4.0. Also, environmental protection research is characterized by a wide variety of research themes and multidisciplinary endeavours, in contrast with industry 4.0 .
\end{abstract}

(c) 2018 Institution of Chemical Engineers. Published by Elsevier B.V. All rights reserved.

\section{Introduction}

The themes of process safety and environmental protection (PSEP) have implications for various areas of production systems (Gysin, 1976). Process safety is the common global language used to communicate the strategies of hazard identification and analysis, risk assessment and evaluation, safety measures, and safe critical decision making (Khan et al., 2015). Another driver to address hazardous installations and major accidents has been the regulations introduced by governments for environmental protection (Hutton, 2017).

Companies' decisions directed towards sustainable production systems will require a consistent and continuous procedure, especially in the PSEP fields. Industry 4.0 has been considered one of the major trends in production systems, with clear implications for sustainability in organizations (Jabbour et al., 2018a). Industry 4.0 is an emerging trend in production systems and is described as a concept that encompasses technologies such as the Internet of Things (IoT) (Lee and Lee, 2015), big data (Lee et al., 2014; Hauhang

\footnotetext{
* Corresponding author.

E-mail address: gobbo@feb.unesp.br (J.A. Gobbo Junior).
}

et al., 2015), cyber-physical systems (CPS) (Lee et al., 2015), smart manufacturing (Kang et al., 2012), and smart objects (Fortino et al., 2013; Gobbo Junior et al. 2017).

Thus, this paper argues that integration among concepts and technologies of industry 4.0 and PSEP would unlock the potential for sustainable production systems. An additional benefit to do research in these subjects would come from the already existing interaction between emerging topics in industry 4.0 and PSEP functions. A resulting cross-fertilization of ideas may facilitate the identification of novel approaches to risk reduction and environmental protection (Hutton, 2017). Therefore, the research in the PSEP arena will need to be integrated in the emerging cutting-edge discussion of industry 4.0.

Despite the fact that process safety and environmental protection researchers have been pioneers in the study of the impact of automation on safety, industry 4.0 will present new challenges and opportunities for process safety and environmental protection researchers. Angell and Klassen (1999) proposed an integration of environmental issues into research in productive systems. Early research integrating industry 4.0 and sustainable production concepts includes the works of Jabbour et al. (2018a, 2018b) and Jabbour et al. (2017). 
Although there are very few studies on the frontier of the relationship between these subjects and systematic reviews on the isolated themes, no studies have attempted to integrate, through a systematic analysis, concepts in PSEP and industry 4.0. Another gap in the literature is the lack of research with a systematic approach in effectively identifying the main concepts related to industry 4.0 and PSEP, as well as the existing intersections among these subjects.

In line with this gap, this paper focuses on identifying and systemizing information regarding the integration of PSEP and industry 4.0 concepts. While pointing out early intersection points between these subjects, we will also address possible directions for future integrative research into these fields, following Jabbour (2013). Consequently the primary question guiding this research is: what are the possible gaps and intersections points between PSEP and industry 4.0 concepts?

Taking this into account, the outline of this paper is to identify emerging research fronts in PSEP and industry 4.0. A secondary aim is to identify possible gaps and intersections as well as new directions in integrating the research themes in PSEP and industry 4.0. This identification of possible gaps and intersections will be elaborated by means of one very specific but influential type of evidence: topological measures in co-occurrences of keywords.

The paper's sections are organised as follows: the main theoretical background on PSEP and industry 4.0 is described in Section 2; approaches for visualization of bibliometric networks and extensions are described in Section 3; the research method is presented in Section 3; the results are discussed in Section 4; and in Section 5 some conclusions are presented about this work.

\section{Theoretical background}

\subsection{Process safety: concepts and principles}

Safety is a relative concept that must be understood in the presence of some hazard or risk. The concept of risk is related to both hazards created by humans and those created by nature; consequently, safety constitutes an ability to reduce or eliminate the likelihood of hazardous events occurring (Antonsen, 2009). The main focus of risk analysis is to support decision making by assessing and quantifying the risks associated with the operation and design of a technical system (Aven, 2016). Risk assessment and risk management provide important contributions in supporting the decision-making process. Therefore, risk assessment and management are a set of principles and methods developed to conceptualize, assess, and manage risk and recognized threats (also called hazards) (Aven, 2016).

A growing interest in the process safety field is the concept of safety culture. The existing research in the field supposes a connection between safety culture and safety. The conceptualization of safety culture is by no means conclusive. Aligned with this view, organizational culture may help to create safety by being a medium to disseminate safety-critical knowledge (Antonsen, 2009). Therefore, the inherent safe concept could be obtained in a process or product design that avoids hazards instead of controlling them. This stage would be achieved through four main methods: minimizing (reducing the amount of hazardous material), substituting (replacing one material with another of less hazard), moderating (using less hazardous process conditions), and simplifying (design process to be less complicated and therefore less prone do failure) (Heikkilä, 1999).

Process safety is identified as an integral part of process development and manufacturing rather than an "add-on" to the process (Gibson, 1999). Process safety differs from occupational safety as it solely focuses on preventing and mitigating major process accidents such as fires, explosions, and toxic releases. Process safety assessment/management includes several essential steps. Though every step is equally important, hazard identification, risk assessment, and management can be considered as the key steps of process safety management (Bahr, 1997). The primary objective of process safety is to prevent the unwanted release of highly hazardous chemicals/biological agents into locations, which could expose humans to serious hazards. Process safety management is an approach to evaluate processes that have the potential to cause catastrophic incidents such as fires, explosions, or toxic releases (OSHA, 2000).

Extensive industrial automation and computer control create many new issues in process safety, which have been extensively discussed in the literature (Hendershot, 2006). This is mainly due to human factors. Examples of different applications of automation to avoid human errors can be found in Hendershot (2006), Cameron et al. (2017), and Taylor (2017), not only to avoid safety hazards but also to automate Hazop's analysis or prevent maintenance.

\subsection{Environmental protection: concepts and principles}

Environmental protection is the practice of protecting the natural environment of the individual, organizational, and governmental levels, for the benefit of both the environment and humans (Kraft, 2017). Traditionally, environmental protection has been considered a public interest and external to the private sphere (Mazurkiewicz, 2004). The idea behind this concept is that the biophysical environment has been degraded and governments have begun to place restraints on activities that cause environmental degradation (White, 2017). Since its popularization, the concept of environmental protection has become closely associated with the concept of sustainable development. However, gradually the focus is shifting from sustainable development to the multiplicity of sustainability and analyses of the paths in which they are shaped (Haughton and Counsell, 2004).

Discussion concerning environmental protection often focuses on the role of government, legislation, and law enforcement. However, in its broadest sense, environmental protection may be seen to be the responsibility of all stakeholders, including industry, environmental, and community groups (Kraft, 2017). Gradually, environmental decision-making processes are evolving to reflect this broad base of stakeholders and are becoming more collaborative in many countries. The stringency of environmental regulations affects eco-innovation as firms respond to stricter environmental regulations with higher levels of eco-innovations (Kesidou and Demirel, 2012).

Peachey (2008) says that environmental protection management involves a complete assessment of the full impacts of power source choices and a conscious decision about what environmental impacts and mitigation measures are going to be made. The terms environmental quality, air quality, soil quality, and water quality invariably connote the status or condition of each condition that relates to requirements of wildlife or to human requirements and/or preferences (Johnson et al., 1997).

These are related to the role of government in environment protection. Urban air pollution poses a significant threat to human health, property, and the environment throughout both the developed and developing parts of the world. The issue of urban air quality is receiving increasing attention as a growing share of the world's population is now living in urban centres and demanding a cleaner urban environment (Gurjar et al., 2008). One impact of air emissions is that they also contain significantly high levels of sulphur components-heavy metals and fly ash being generally of greater regional concern because of direct health and environmental impacts (Peachey, 2008). All of them need to be monitored to ensure that they are in control under the specifications of govern- 
ment institutions. There are different technology devices to this control.

Analysing the subject of environmental protection automation has been used to control water quality, air quality, and heavy metals (Zhang et al., 2016; Mishra et al., 2011), among other monitoring required to meet the specifications of government. One application of data automation in the environmental protection field is the data mining approach. Nawahda (2016) applied data mining for predicting surface ozone levels with traffic information.

\subsection{Industry 4.0: concepts and technologies}

Industry 4.0 is a promising approach based on integration of manufacturing and business processes in the company's own operation and supply chain. Technical aspects of these requirements are addressed by the application of the generic concepts of CPS, the IoT, cloud computing, big data, smart objects, and manufacturing to the industrial production systems (Lee and Lee, 2015; Lee et al., 2014; Hauhang et al., 2015; Lee et al., 2015; Kang et al., 2012; Fortino et al., 2013; Gobbo Junior et al. 2017).

CPS are defined as the joint work of computers and physical systems, which allow the integration between physical processes and objects in order to connect machines and devices in a network of production lines (Lee and Lee, 2015; Lee et al., 2015). Technically, CPS consist of four main elements: the cybernetic elements (computers and software) that enable physical elements to become smart objects; the computational networks that allow smart objects to communicate with each other and with the environment; sensors and actuators embedded in smart objects; and the environmental capture and transmission information (Gunes et al., 2014).

The IoT refers to the interconnectivity of physical objects and the Internet through wireless or cable networks and the use of unique identification codes for the purpose of mutual exchange of data (Atzori et al. 2010, Gubbi et al. 2013). As a consequence, the IoT depends on a number of technologies (Lee and Lee, 2015): identification technologies such as radio-frequency identification (RFID), which allow the identification of objects and automatic data capture; wireless network sensors to monitor physical and environmental conditions as well as provide information about objects such as their location and movements; middleware that allows mediation between software and other applications, moving or transforming information between different platforms, communication protocols, and operating systems; cloud computing for the processing and storage of a large amount of data generated in the organization; and application software that enables robust data transmission and interaction between objects and people.

These smart production systems are implemented with the objective of creating a horizontal integration of digital networks and a vertical integration of these networks with the manufacturing systems (Kagermann et al. 2011, Prause, 2015, Strandhagen et al., 2016). Smart manufacturing systems objectives include the development of CPS and dynamic production networks with the objective of building up flexible and open value chains in the manufacturing of mass customization products (Ramsauer, 2013). Five characteristics are associated with the term, namely context awareness, modularity, heterogeneity, interoperability, and compositionality (Mittal et al., 2017). The smart factory concept also describes smart production systems that operate in the environment of a factory, while they are able to connect with a global network of similar production systems (Mittal et al., 2017).

\section{Research method}

Bibliometric networks are composed of nodes-publications, journals, researchers, or keywords-and edges, which indicate relations between pairs of nodes (Van Eck and Waltman, 2014). Once referred to as 'science mapping', bibliometric networks have been admitted to the mainstream of bibliometric research (Shibata et al., 2008). Many different approaches have been proposed for visualizing bibliometric networks. The most commonly studied types of bibliometric network relations are citation, co-citation, bibliographic coupling, keyword co-occurrence, and co-authorship. Popular visualization techniques include distance-based, graphbased, and timeline-based approaches (Van Eck and Waltman, 2014).

Our focus in this research is on the co-occurrence network approach. Networks of co-occurrences of keywords have also been studied extensively (Luiz et al., 2016). Keywords of a publication can be extracted from the author-supplied keyword list, or from even the title or abstract (Van Eck and Waltman, 2014). In some cases, especially in the older literature, keywords are restricted to individual words, but in other cases they also include terms consisting of multiple words. For examples of early work on keyword co-occurrence networks, we refer to Callon et al. (1983) and Peters and Van Raan (1993).

Google Scholar database provides a broader coverage in comparison with Scopus and ISI Web of Science (Harzing and Alakangas, 2016). Scopus database therefore is more extensive than ISI Web of Science, however in the later, Natural Sciences and Engineering (NSE) journals are well overrepresented (Mongeon and Paul-Hus, 2016). NSE is an important discipline for PSEP and industry 4.0 researchers and have the biggest share of journals in ISI Web of Science in comparison with Scopus.

The source of the keyword data used for this research was the ISI Web of Science database, which differs from others by its wide content and by grouping an updated database, which includes articles in the preview phase and very recent publications. The data were collected in November 2017 by using a keyword search in the database search engine. The keywords selected were "industry 4.0", "environmental protection" and "process safety". The selection fields considered were Title, Abstract, and Keywords, with no restrictions of period, language, journals, or type of documents. To explore the link between the themes, bibliographic data were downloaded for all publications and are listed in Table 1 . The ISI Web of Science database supports various file formats. We used the text (txt) format to capture details of all documents, and the bibliographic data were downloaded in batches of 500 publications at each time (Table 1). Therefore, we downloaded three batches for "industry 4.0", 58 batches for "environmental protection", and four batches for "process safety". A previous search, linking all the three selected keywords, was conducted in VOSviewer and for the combination "Environmental Protection" and "Process Safety" and "Industry 4.0" there are no records. The analysis has been done by topics, which contemplate title, abstract and keywords. This search has been done at SCOPUS database, as also and there are no documents for this combination of keywords. Therefore, we suppose that an individual selected keyword search highlights the keywords

Table 1

Data collected from Web of Science.

\begin{tabular}{cllc}
\hline & \multicolumn{2}{l}{ Web of Science } & \\
\cline { 2 - 4 } $\begin{array}{c}\text { Keywords used in this } \\
\text { research }\end{array}$ & Industry 4.0 & $\begin{array}{l}\text { Environmental } \\
\text { Protection }\end{array}$ & Process Safety \\
\hline $\begin{array}{c}\text { Documents identified } \\
\text { with the keywords } \\
\text { Number of Keywords }\end{array}$ & 789 & 28879 & 1873 \\
\hline
\end{tabular}




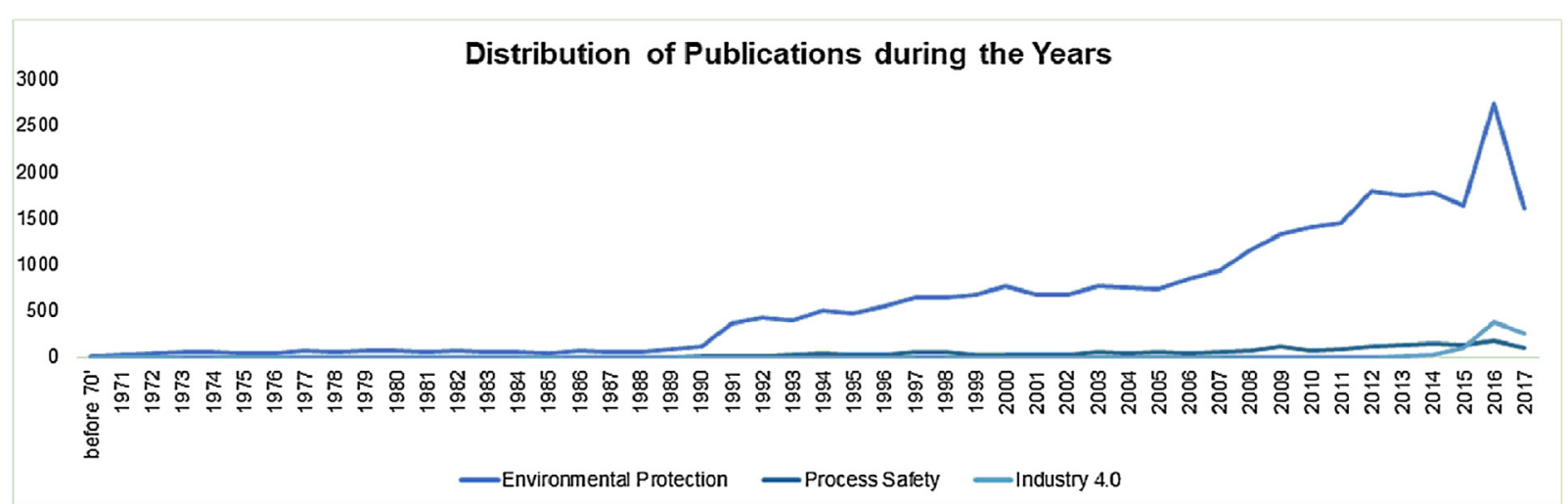

Fig. 1. Distribution of publications of Environmental Protection, Process Safety and Industry 4.0.

co-occurrence network for each topic. The missing literature for this combination of selected keywords helps to justify the need to identify possible gaps and intersections points between PSEP and industry 4.0 concepts.

After the collection of data, the database in text extension was entered in VOSviewer to analyse the co-occurrence of keywords. The search for keywords co-occurrence was referenced to the title and abstract, with a minimum of five co-occurrences and with binary counting, disregarding repetitions. VOSviewer has some special text-mining features and also allows editing, correcting errors, and adding missing bibliographic data elements. Therefore, we also applied a filter available in VOSviewer to not consider irrelevant terms such as articles and prepositions. After the selection by the software, the least relevant items were excluded and manual filtering was used to exclude words considered not relevant (for example: findings, conclusions, etc.).

As a limitation, this software treats direct citations relations as undirected relations; however, mapped networks may consist of several thousand nodes. To construct co-occurrence networks of terms, VOSviewer uses text-mining functionality to extract textual data from titles and abstracts of publications (Van Eck and Waltman, 2014). The VOSviewer filter selects all word sequences and plural noun phrases and converts them into singular ones. Therefore, the software calculates for each noun phrase a relevance score (Van Eck and Waltman, 2014). To identify similarities between keywords in PSEP and industry 4.0, spreadsheet software was used. A list of keywords was built considering each period and its respective clusters.

\section{Results and discussion}

The initial analysis is about the distribution of publications presented in Fig. 1. This figure indicates an incipient number of articles related to the topic in the first years, growing significantly in recent years.

There is a more interest in the early 1990s for this theme, probably due to the world meetings and climate conferences on the environment, like the conferences in Toronto in 1988, Geneva in 1990, and Rio de Janeiro in 1992, which placed the theme on global agendas. Environmental protection is a theme that has been explored since 1970; however, industry 4.0 is a recent theme, for which a first publication was dated 2012, and process safety has shown a strong presence in academic research since 1991.

The graphical analysis of VOSviewer depicted the researchers' country origin network related to each keyword. Figs. 2-4 show the main countries and the links between the researchers in different universities. In the visualization presented in Figs. 2-4, each circle represents a country. Large circles represent countries that have a large number of publications. Small circles represent countries with fewer publications. In general, the closer that two countries are located to each other in the visualization, the more strongly they are related to each other based on bibliographic coupling. In other words, countries that are located close to each other tend to cite the same publications, while countries that are located far away from each other usually do not cite the same publications. Colours indicate clusters of countries that are relatively strongly related to each other.

Analysing Fig. 2, it is possible to visualize that in the environmental protection field the countries that have larger influence were the U.S. and China. Already in the case of research about process safety the country that develops more research about this subject is the U.S. (Fig. 3), and it is possible to say that England, China, Germany, and Canada are emerging forces and have strong influence along with the U.S. Finally, research about industry 4.0 started in Germany, which together with China remains the main country that has researched this subject (Fig. 4).

\subsection{Co-occurrence network of terms (keywords)}

In the visualization presented in Figs. 5-7, keywords networks were grouped into clusters. In the cluster of environmental protection (Fig. 5), 24 keywords clusters were identified, with the largest one related to water and air quality and heavy metals. The second large cluster of keywords is related to environmental management and the third with hazards identification and assessment. It is a dense network comprising more than 500 keywords.

In the cluster of process safety (Fig. 6), 12 keyword clusters were identified, with the largest one related to fault tree analysis; the following clusters were auditing, decision making, chemical process, and inherent safety design. This network has a smaller density in comparison with environmental protection and comprises 124 keywords. The higher density of the environmental protection keywords network could be explained by the larger number of papers published yearly (589 papers per year) since the 1970 s, in comparison with process safety (38 papers per year). Because of the much newer history of industry 4.0, with the first paper published in 2012, it has a more sparsely connected keyword network and comprises 62 keywords. PSEP also has a smaller number of clusters (10); the first cluster is related to CPS and automation, the second to manufacturing technologies, and the third to digitalization issues.

Tables 2-4 show the result of keyword co-occurrence searches for the 10 main words (by number of occurrences) and the total link strength for each keyword. It is possible to observe that between the most co-occurrence keywords in PSEP and industry 4.0 there are no similarities.

The analysis of similarities shows that there are more intersections between environmental protection and industry 4.0 (19 keywords co-occurrences) than with process safety (6 keywords 


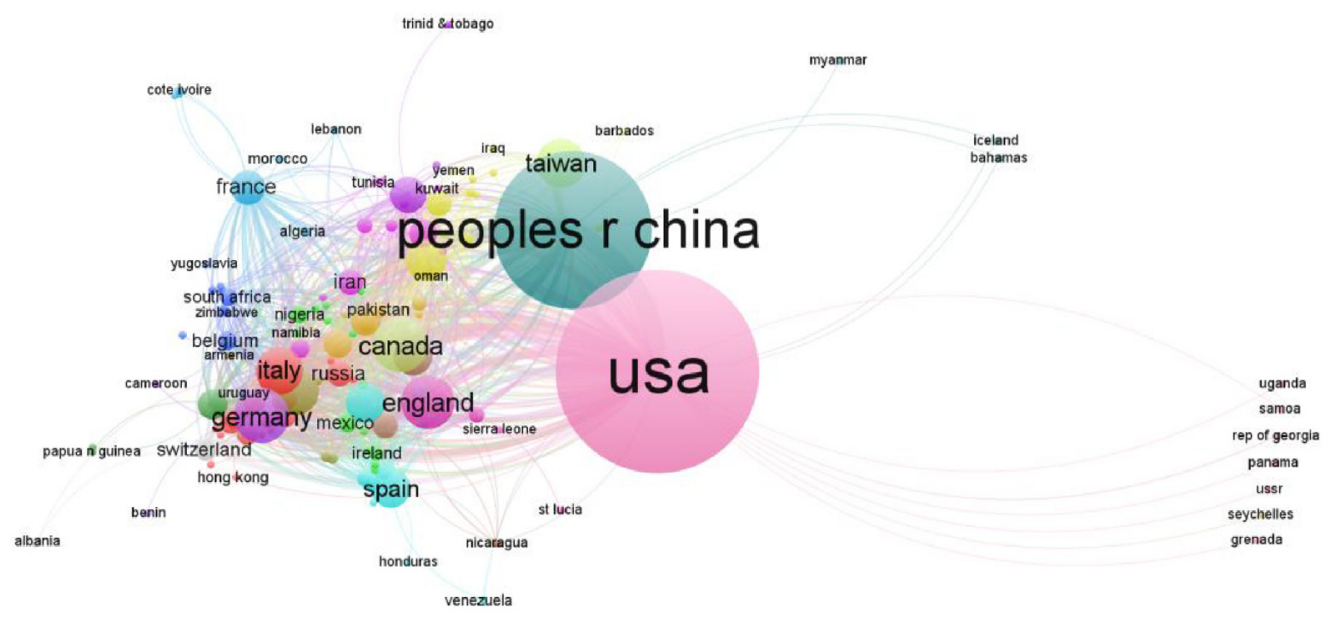

Fig. 2. Researcher country network - Environmental Protection.

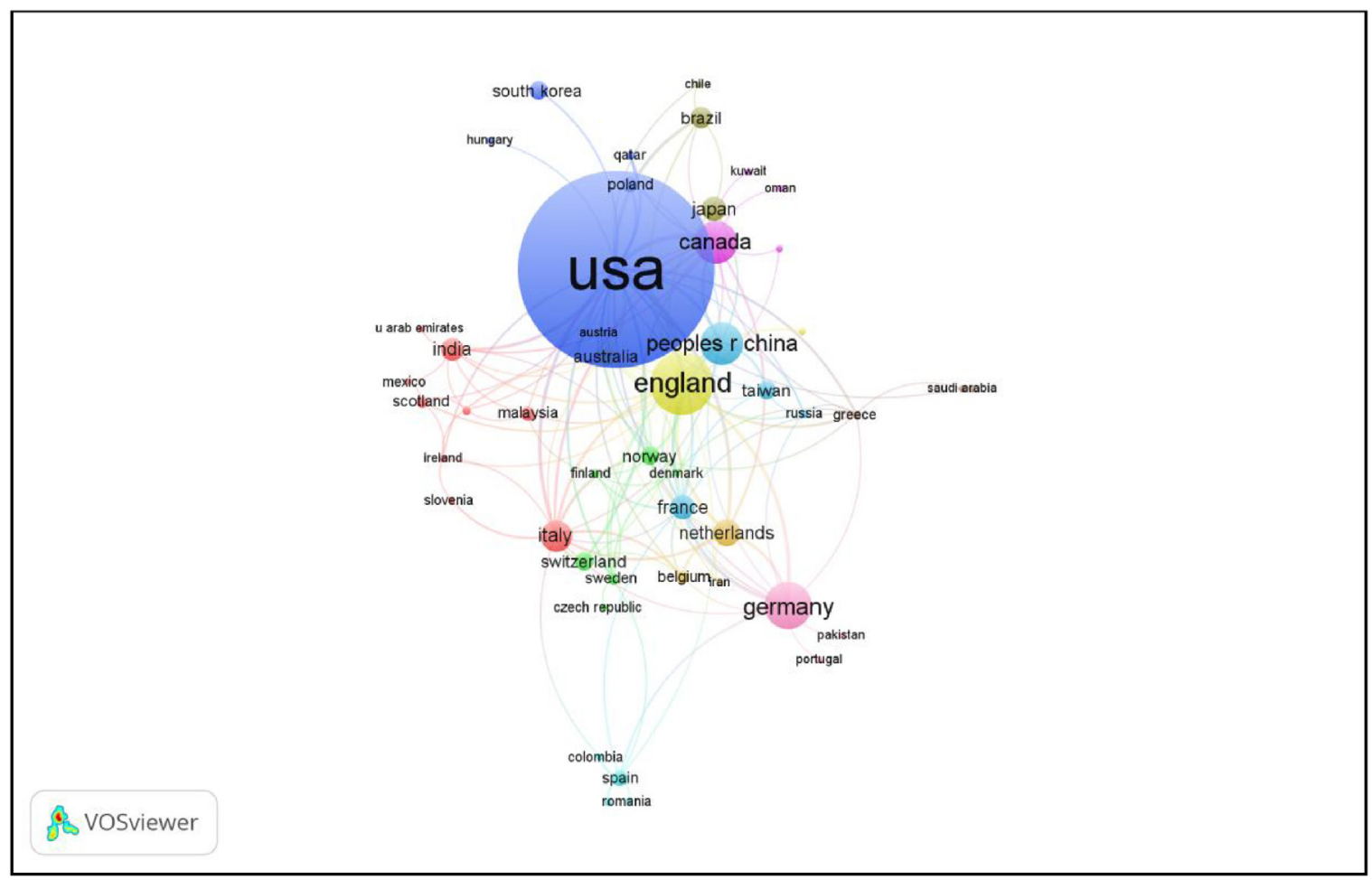

Fig. 3. Researcher country network - Process Safety.

co-occurrences). Possible gaps were identified in Table 5 and are composed of the non-existent intersection between keywords co-occurrences in industry 4.0, process safety and environmental protection. There are also similarities between the following PSEP and industry 4.0 keywords in Table 5: automation; integration, maintenance, optimization and simulation. Most of the papers using this combination of keywords in industry 4.0 and PSEP were published in English, and country authors were mainly from China, Germany and USA. Documents for industry 4.0 and process safety were classified mainly in the engineering, computer science and automation control systems fields. Environmental protection has a more diverse documentś classification (proceeding papers, 


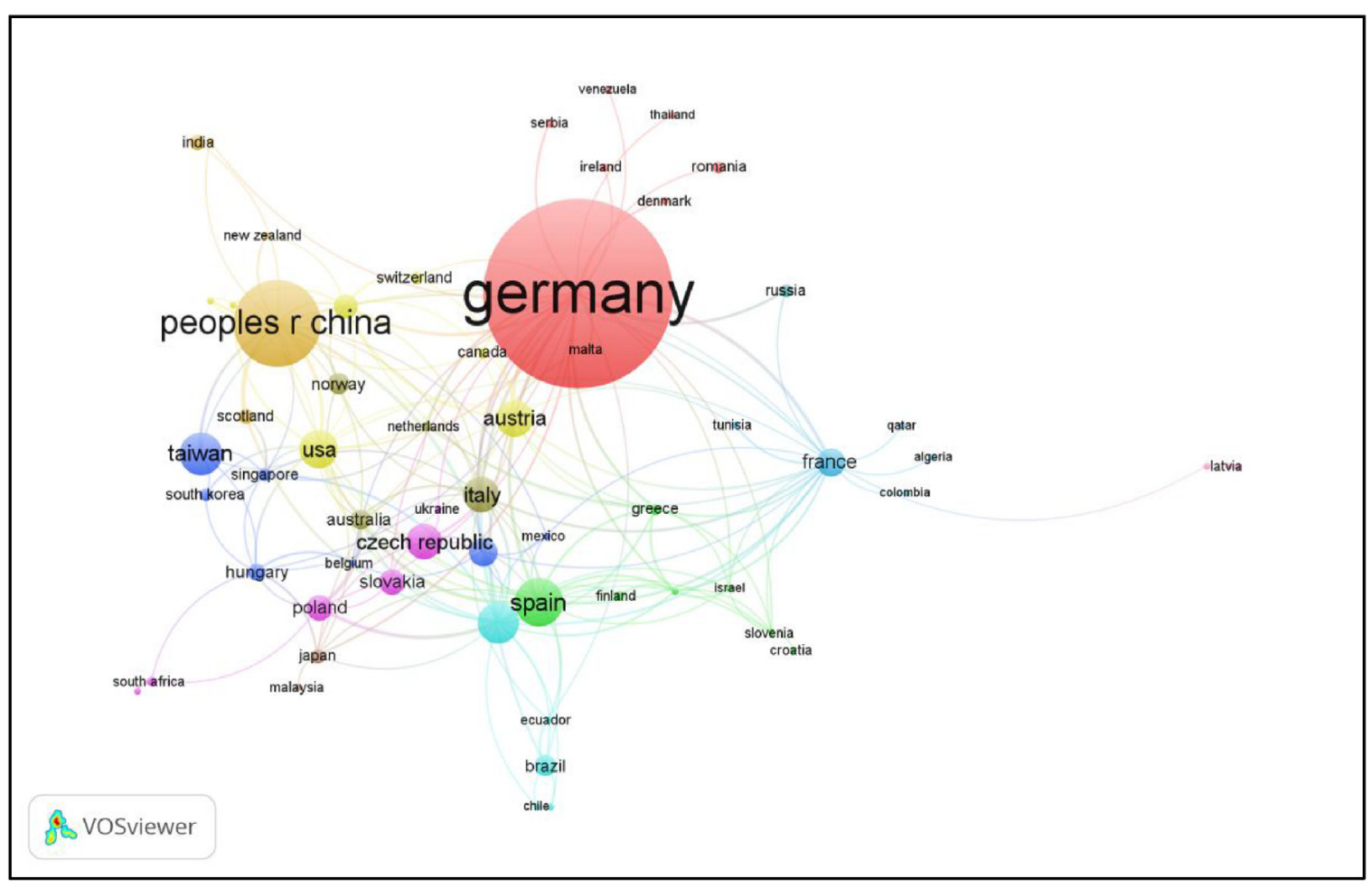

Fig. 4. Researcher country network - Industry 4.0 .

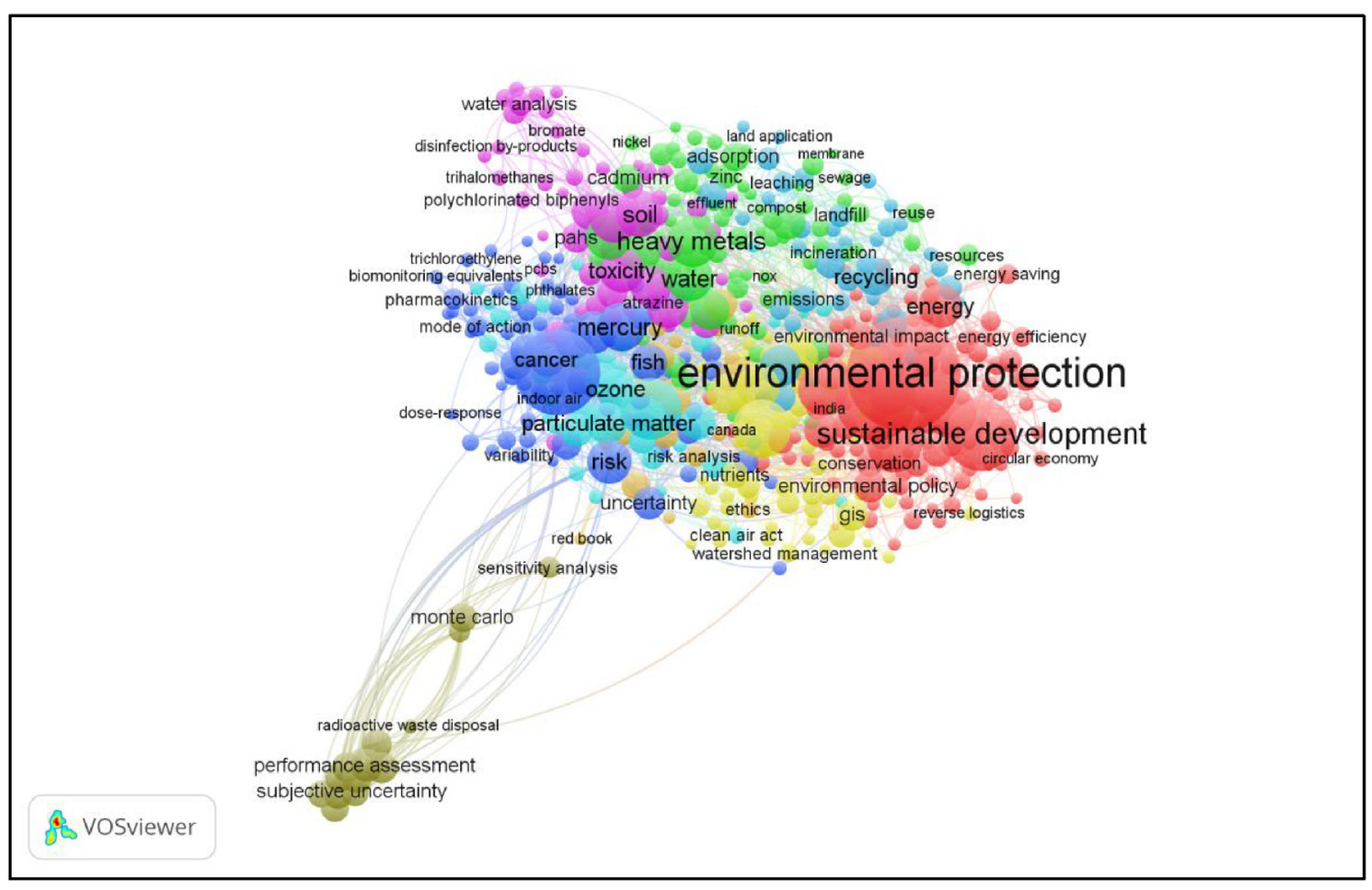

Fig. 5. Keywords and cluster by color for the search of "Environmental Protection".

articles, reviews, book chapter, etc.) and fields (engineering, chemistry, materials science, environmental science ecology, computer science, water resources and energy fuels). Probably this result was due to the higher number of articles and keywords observed in environmental protection in comparison with the other clusters (industry 4.0 and process safety). Therefore, environmental protection reveals more diverse application of the similar keywords (automation, integration, maintenance, optimization and simulation) and greater possibilities of application of industry 4.0 concepts, due to this multidisciplinary characteristic.

Linking concepts between these arenas would provide new directions for research. One example, one would be to explore the 


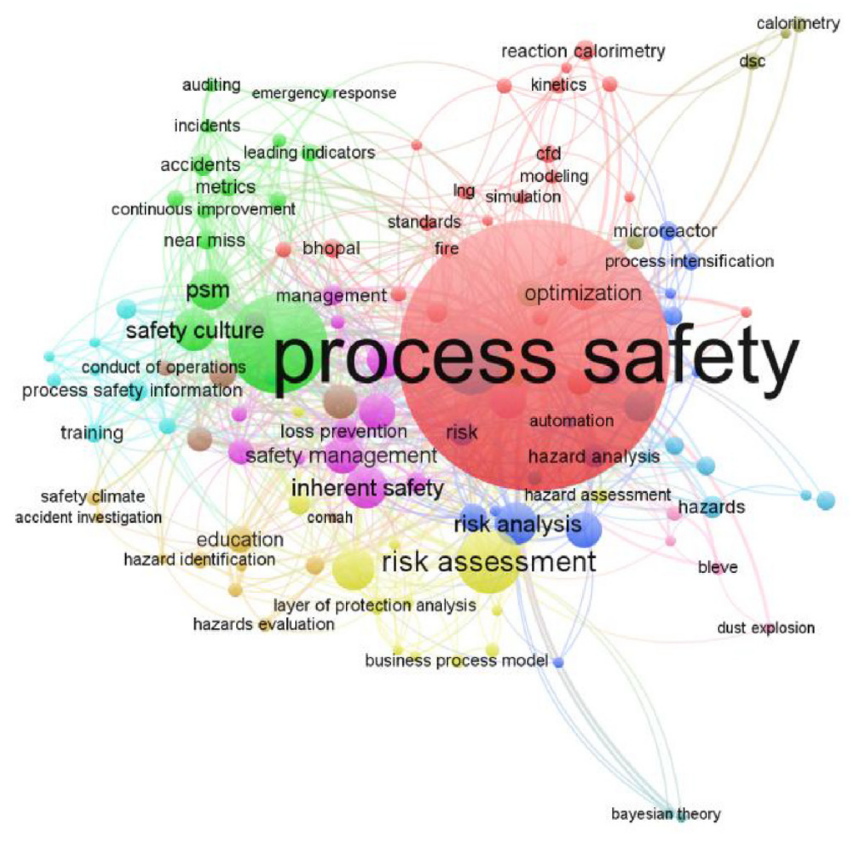

Fig. 6. Keywords and cluster by color for the search of "Process Safety".

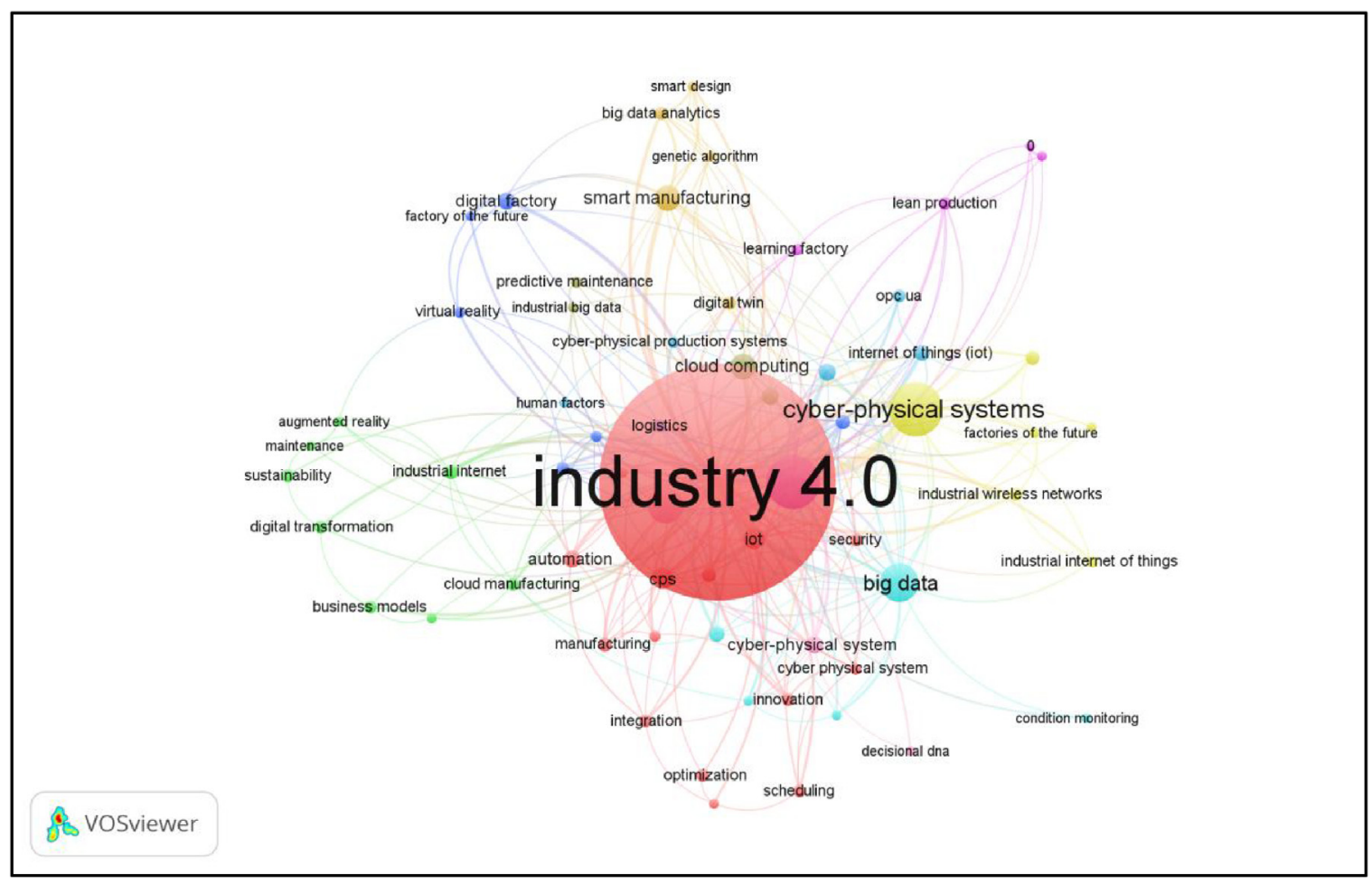

Fig. 7. Keywords and cluster by color for the search of "Industry 4.0".

use of information technology, especially cloud computing and data mining, in the process safety field. Cloud computing would support organizations in storing large amounts of data, related to risk assessment, analysis, and management activities and applications in the cloud. Organizations could also improve their process safety by using data mining techniques and genetic algorithms in the large amount of safety-related data stored in the cloud for pattern discovery and solution optimization. Although we have not found in our research a relationship between the process safety cluster and the keyword data mining, Qi et al. (2012) and Cameron et al. (2017) showed that this tool can be a way to organize data, find patterns, and propose changes to anomalies by developing an active 
Table 2

10 main Keywords of search "Environmental Protection".

\begin{tabular}{lll}
\hline Keyword & Occurences & Total Link Strength \\
\hline environmental protection & 1509 & 2225 \\
sustainable development & 521 & 899 \\
risk assessment & 445 & 1104 \\
environment & 442 & 1021 \\
china & 296 & 560 \\
air pollution & 269 & 605 \\
sustainability & 230 & 470 \\
water quality & 227 & 543 \\
heavy metals & 209 & 435 \\
mercury & 160 & 388 \\
Total Clusters & & $\mathbf{2 4}$ \\
\hline
\end{tabular}

Table 3

10 main Keywords of search "Process Safety".

\begin{tabular}{lll}
\hline Keyword & Occurrences & Total Link Strength \\
\hline process safety & 318 & 358 \\
process safety management & 83 & 137 \\
risk assessment & 46 & 75 \\
safety & 43 & 65 \\
process hazard analysis & 27 & 46 \\
safety culture & 26 & 51 \\
risk analysis & 26 & 44 \\
inherent safety & 26 & 37 \\
psm & 25 & 63 \\
risk management & 22 & 31 \\
Total Clusters & & $\mathbf{1 2}$
\end{tabular}

Table 4

10 main Keywords of search "Industry 4.0".

\begin{tabular}{lll}
\hline Keyword & Occurrences & Total Link Strength \\
\hline industry 4.0 & 359 & 388 \\
internet of things & 50 & 99 \\
cyber-physical systems & 49 & 92 \\
big data & 31 & 61 \\
smart factory & 28 & 56 \\
smart manufacturing & 18 & 39 \\
cloud computing & 18 & 34 \\
iot & 15 & 33 \\
cps & 14 & 31 \\
industrie 4.0 & 11 & 17 \\
Total Clusters & & $\mathbf{1 0}$ \\
\hline
\end{tabular}

Table 5

The similarities of words among the three clusters.

\begin{tabular}{llll}
\hline $\begin{array}{l}\text { Similarities of } \\
\text { Keywords }\end{array}$ & $\begin{array}{l}\text { Cluster Industry } \\
4.0\end{array}$ & $\begin{array}{l}\text { Cluster } \\
\text { Environmental } \\
\text { Protection }\end{array}$ & $\begin{array}{l}\text { Cluster Process } \\
\text { Safety }\end{array}$ \\
\hline automation & $\mathrm{x}$ & $\mathrm{x}$ & $\mathrm{x}$ \\
cloud computing & $\mathrm{x}$ & $\mathrm{x}$ & not exist \\
data mining & $\mathrm{x}$ & $\mathrm{x}$ & not exist \\
genetic algorithm & $\mathrm{x}$ & $\mathrm{x}$ & not exist \\
human factors & $\mathrm{x}$ & not exist & $\mathrm{x}$ \\
information & $\mathrm{x}$ & $\mathrm{x}$ & not exist \\
$\quad$ technology & & & \\
innovation & $\mathrm{x}$ & $\mathrm{x}$ & not exist \\
integration & $\mathrm{x}$ & $\mathrm{x}$ & $\mathrm{x}$ \\
internet of things & $\mathrm{x}$ & $\mathrm{x}$ & not exist \\
logistics & $\mathrm{x}$ & $\mathrm{x}$ & not exist \\
maintenance & $\mathrm{x}$ & $\mathrm{x}$ & $\mathrm{x}$ \\
manufacturing & $\mathrm{x}$ & $\mathrm{x}$ & not exist \\
optimization & $\mathrm{x}$ & $\mathrm{x}$ & $\mathrm{x}$ \\
rfid & $\mathrm{x}$ & $\mathrm{x}$ & not exist \\
security & $\mathrm{x}$ & $\mathrm{x}$ & not exist \\
simulation & $\mathrm{x}$ & $\mathrm{x}$ & $\mathrm{x}$ \\
smart grid & $\mathrm{x}$ & $\mathrm{x}$ & not exist \\
supply chain & $\mathrm{x}$ & $\mathrm{x}$ & not exist \\
supply chain & $\mathrm{x}$ & $\mathrm{x}$ & not exist \\
management & & $\mathrm{x}$ & not exist \\
sustainability & $\mathrm{x}$ & & \\
\hline
\end{tabular}

knowledge-based retrieval system that provides users necessary information about chosen chemicals by integrating data mining with text mining to record and analyse the incidents. This data storage can also be done by cloud computing and can be optimized by genetic algorithms.

The human factor concept is concerned with the alignment between the user, equipment, and the environment. Despite extensive studies within process safety for the role that human factors have on safety, there is a current gap in research in relation to environmental protection.

Adoption of innovations in the PSEP field, such as the IoT and technologies related to security (safeguarding of personal data) should be further explored. Fabiano (2017) affirmed that future research in innovation is required to cope with new challenges and connected safety. The use of sensors connected to the Internet to monitor physical and environmental conditions would allow manufacturing organizations to proactively and effectively reduce equipment and environment-related hazards. Logistic routes would be optimized according to safety and environmental indicators with RFID support. The smart grid concept involves the use of an improved information system to match energy consumption with production (Gobbo Junior et al., 2017) and could reduce the risk associated with equipment failure or malfunctioning. These initiatives of integration between industry 4.0 and PSEP concepts could be expanded from the organization perspective to the supply chain, therefore leading to progress towards sustainability.

By constructing the matrix in Table 6 we relate and propose potential connections between the main keywords (by number of co-occurrences) of PSEP with industry 4.0. An explanation of the relationships of these keywords follows Table 6 . The number of keywords used in Tables 2-4 was reduced; excluded keywords included acronyms (e.g., IoT) or words in different languages with the same meaning (e.g., industrie 4.0).

Process safety management (PSM) would benefit from the IoT and CPS. These technologies could help to avoid accidents and prevent future loss through the collection of data from processes and objects, such as manufacturing equipment, being possible to identify potential sources of hazard failures. Big data and cloud computing technologies would be beneficial to PSM by applying a large amount of data to plan, monitor, and control variables related to human factor errors and hazards of industry equipment.

The set of principles and methods of risk assessment, analysis and management (process safety), and risk assessment (environmental protection) would benefit from sensors and RFID tags in smart objects informing operators of risks related to materials or components in manufacturing environments. Risk assessment could be more assertive with the technology of data mining, cloud computing, and the IoT, because it is possible to use historical data to give more confidence to predictions related to probability and potential loss. The IoT and CPS would provide similar benefits to risk assessment and analysis as the ones described in PSM.

The process hazard analysis approach could be improved through the use of both cloud computing and big data, because these technologies would allow the collection, storage, sharing, and fast processing of large amounts of data. Cameron et al. (2017) affirmed that to improve the effectiveness of hazard identification and scenario definition, a systems approach and a computer supported/semi-automated methodology is paramount. Sensors embedded in objects could obtain hazard data and transmit information through RFID. This information would be accessed in real time, to improve and accelerate the decision-making process.

The concepts of safety, safety culture, and inherent safety could especially benefit by two main characteristics associated with smart manufacturing systems: awareness and interoperability. Increasing safety awareness in manufacturing operations could be a beneficial consequence of this concept adoption. Interoperability 
Table 6

Matrix of relationships between the main Keywords of Process Safety, Environmental Protection and Industry 4.0.

\begin{tabular}{|c|c|c|}
\hline Process Safety & & Industry 4.0 \\
\hline Process Safety Management & AND & Big Data; Cloud Computing; Internet of Things; Cyber Physical Systems \\
\hline Risk Assessment; Risk Analysis; Risk Management & & Big Data; Cloud Computing; Internet of Things; Cyber Physical Systems \\
\hline Process Hazard Analysis & & Big Data; Cloud Computing; Internet of Things; Cyber Physical Systems \\
\hline Safety Culture; Safety; Inherent Safety & & Smart Factory; Smart Manufacturing \\
\hline Environmental Protection & & Industry 4.0 \\
\hline Sustainable Development; Sustainability & AND & Smart Factory; Smart Manufacturing \\
\hline Risk Assessment & & Big Data; Cloud Computing; Internet of Things; Cyber Physical Systems \\
\hline Environment & & Big Data; Cloud Computing; Internet of Things; Cyber Physical Systems \\
\hline Air Pollution; Water Quality; Heavy Metals; Mercury & & Big Data; Cloud Computing; Internet of Things; Cyber Physical Systems \\
\hline China & & $\begin{array}{l}\text { Big Data; Cloud Computing; Internet of Things; Cyber Physical Systems; Smart } \\
\text { Factory; Smart Manufacturing }\end{array}$ \\
\hline
\end{tabular}

would reduce failures in systems operations interfaces, reducing potentially related hazards, accidents, or material losses and making the production systems more inherently safe.

The sustainable development and sustainability concepts could be improved by the smart factory and manufacturing systems. Smart production systems in the environment of a factory or a network create horizontal and vertical integration of digital and manufacturing systems, building up flexible and open systems. These systems are more oriented to services, instead of physical products, therefore relying less on material usage. Less environmental impact would be a result, as also would be a more distributed manufacturing system, therefore reducing $\mathrm{Co} 2$ transport-related emissions.

The environment could be controlled from the use of IoT technologies, from sensors to apps, in order to plan, monitor, and control environmental factors related to land management between harvesting rotations (Jabbour et al., 2018b), the application of IoT-related technologies to develop smart cities concepts in urban environments (Kehua et al., 2011; Su et al., 2011), uninterrupted monitoring of environmental changes and precision location of pollution sources (Lu et al., 2015).

The use of big data and cloud computing would optimize processes and improve information gathering to predict and better control water quality, air pollution, and contamination by heavy metals or mercury. Automation has been used to control water quality, air quality, and heavy metals (Zhang et al., 2016). Nawahda (2016), for example, applied data mining for predicting surface ozone levels with traffic information. However, sensors and RFID technology embedded in smart objects connected to the Internet would efficiently collect information and allow control of environmental conditions in real time. Artificial intelligence embedded in CPS would allow automating the decision-making process, therefore leading to rapid decision making in the case of potential threats in production systems.

Increasing attention has been paid to urban and agricultural soil pollution with heavy metals in China (Dong et al., 2018). Mining is considered to be one of the most significant sources of heavy metal contamination in China (Li et al., 2014). China's pesticide industry was ranked the second largest in the world. The adoption of industry 4.0 concepts and technologies in China, such as big data (Lu et al., 2015) or smart manufacturing, could lead to improved environmental conditions in that country, specifically the quality of soil, among other problems.

Thus, we further suggest propositions for future studies that integrate the concepts and technologies of industry 4.0 and PSEP topics.

\section{Final remarks}

The results from this research indicate that there are more intersections between the keywords co-occurrences clusters of environmental protection and industry 4.0 than process safety. The intersections between environmental protection and industry 4.0 concepts and technologies are in the fields of supply chain management, technology, production, and human safety and sustainability.

New avenues for research could emerge from the combination of non-existing links between the main keywords in the clusters of environmental protection, process safety, and industry 4.0. It can be concluded that there is more collaborative research in environmental protection and industry 4.0 than with process safety. Also, environmental protection research is characterized by a wide variety of research themes and multidisciplinary work, in contrast with industry 4.0. Geographical inequality in the publication output is identified as a point of concern, because a geographical concentration was observed, mainly in process safety and industry 4.0.

\subsection{Contributions of the work}

This work contributes to integration between PSEP and industry 4.0 concepts and technologies. The main outputs of this research are (a) the matrix of the relationships between the main keywords in PSEP and industry 4.0, where potential connections were identified and discussed between the main keywords (by number of co-occurrences) of PSEP with industry 4.0; and (b) the analysis of similarities of intersections between PSEP and industry 4.0, where possible gaps were identified by the non-existent intersection between keywords co-occurrences.

\subsection{Implications for theory and practice}

The literature has highlighted the benefits from industry 4.0 concepts and technologies adoption for organizations (Geissbauer et al., 2016), logistics activities (Shang et al. 2013), sustainable operations (Jabbour et al., 2018b), and sustainable concepts (Jabbour et al., 2018a; Jabbour et al., 2017). Angell and Klassen (1999) have argued about the necessity of academic works integrating emerging concepts and technologies of productive systems into environmental issues. However, comprehensive literature reviews in industry 4.0 and PSEP concepts integration have not been addressed. Early research integrating industry 4.0 and sustainable production concepts include the works of Jabbour et al. (2018a, 2018b) and Jabbour et al. (2017). Furthermore, the work presents a set of potential connections between PSEP and industry 4.0, as well as existing keywords similarities. There are opportunities to advance knowledge into these fields, by integration of industry 4.0 concepts and technologies into the PSEP field.

\subsection{Managerial implications}

This work presents a set of contributions to managers interested in integrating industry 4.0 concepts and technologies into PSEP. The integration between the explored relationships suggests directions of potential integrations between the concepts and technologies of industry 4.0 and PSEP. The proposed matrix as well 
as the identification of keywords intersections gives to managers practical insights on the adoption of the concepts and technologies of industry 4.0 in PSEP fields. This would lead managers toward the adoption of strategies for digitalization of operations. The matrix allows managers to foresee potential applications of concepts and technologies of industry 4.0 in PSEP, considering a micro (factory) and macro level (productive systems), and examples of applications were provided that would lead to innovations into these fields.

\subsection{Research limitations}

The main idea of bibliometric network visualization is to allow large amounts of complex bibliographic data to be analysed by visualizing core aspects of data. Loss of information takes place when textual data is reduced to a co-occurrence network of terms. Loss of information is especially problematic because it may affect the conclusions that can be drawn from a bibliometric network visualization.

Given the above difficulties, it is useful to visualize a bibliometric network as a complement to rather than as a substitute for expert judgment. Bibliometric network visualizations are most useful when they are interpreted by experts. Another aspect is that for a small amount of data, there often is no benefit in the use of visualization, because it would be better to directly study the available data. Qualitative studies are suggested in order to understand adoption and integration issues related to identified concepts and technologies in PSEP fields.

\section{Acknowledgement}

This research was supported by UNESP - Sao Paulo State University and CAPES - Coordination for the Improvement of Higher Education Personnel - PNPD grant. We had received a helpful input from John Mugge (Proof-Reading-Service.com, Devonshire, UK). We also thank the anonymous reviewers and the editors for their feedback.

\section{Appendix A. Supplementary data}

Supplementary data associated with this article can be found, in the online version, at https://doi.org/10.1016/j.psep.2018.05.017.

\section{References}

Angell, L.C., Klassen, R.D., 1999. Integrating environmental issues into the mainstream: an agenda for research in operations management. J. Oper. Manag. 17 (5), 575-598.

Antonsen, S., 2009. Safety Culture: Theory, Methods and Improvement. Taylor \& Francis, Boca Raton

Atzori, L., Iera, A., Morabito, G., 2010. The internet of things: a survey. Comput. Netw. 54 (15), 2787-2805.

Aven, T., 2016. Risk Assessment and Risk Management: review of recent advances on their foundation. Eur. J. Oper. Res. 253 (1), 1-13.

Bahr, N., 1997. System Safety Engineering and Risk Assessment: a Practical Approach, first ed. Taylor \& Francis Group, Washington.

Callon, M., Courtial, J.P., Turner, W.A., Bauin, S., 1983. From translations to problematic networks: an introduction to co-word analysis. Soc. Sci. Inf. 22 (2), 191-235.

Cameron, I., Mannan, S., Németh, E., Park, S., Pasman, H., Rogers, W., Seligmann, B., 2017. Process hazard analysis, hazard identification and scenario definition: are the conventional tools sufficient, or should and can we do much better? Process Saf. Environ. Prot. 110, 53-70.

Dong, R., Jia, Z., Li, S., 2018. Risk assessment and sources identification of soil heavy metals in a typical county of Chongqing Municipality, Southwest China. Process Saf. Environ. Prot. 113, 275-281.

Fabiano, B., 2017. Editorial: loss prevention and safety promotion in the process industries: issues and challenges. Process Saf. Environ. Prot. 110, 1-4.

Fortino, G., Guerrieri, A., Lacopo, M., Lucia, M., Russo, W., 2013. An agent-based middleware for cooperating smart objects, Commun. Comput. Inf Sci.,387-398.

Geissbauer, R., Vedsø, J., Schrauf, S., 2016. A Strategist's Guide to Industry 4.0: Global Businesses Are About to Integrate Their Operations into a Seamless Digital Whole, and Thereby Change the World. Strategy + business, pp. 83.
Gibson, N., 1999. Process safety-A subject for scientific research. Trans. IChemE 77, $153-179$.

Gobbo Jr., J.A., De Souza, M.G.Z.N., Gobbo, S.C.O., 2017. Barriers and challenges to smart buildings' concepts and technologies in Brazilian social housing projects. Int. J. Sustain. Real Estate Const. Econ. 1 (1), 31-50.

Gubbi, J., Buyya, R., Marusic, S., Palaniswami, M., 2013. Internet of Things (IoT): A vision architectural elements, and future directions. Future Gener. Comput. Syst. $29,1645-1660$

Gunes, V., Peter, S., Givargis, T., Vahid, F., 2014. A survey on concepts, applications and challenges in Cyber-Physical Systems, KSII Trans. Internet Inf. Syst. 8 (12), 4242-4268.

Gurjar, B.R., Butler, T.M., Lawrence, M.G., Lelieveld, J., 2008. Evaluation of emissions and air quality in megacities. Atmos. Environ. 42, 1593-1606.

Gysin, H., 1976. Environmental-protection and industry. Fresenius Z. Anal. Chem. 282 (4), 389-394.

Harzing, A.W., Alakangas, S., 2016. Google Scholar, Scopus and the Web of Science: a longitudinal and cross-disciplinary comparison. Scientometrics 106, 787-804.

Haughton, G., Counsell, D., 2004. Regions and sustainable development: regional planning matters. Geogr. J. 170 (2), 135-145.

Hauhang, G.Q., Zhong, R.Y., Ysui, K.L., 2015. Special issue on big data for service and manufacturing supply chain management. Int. J. Prod. Econ. 165, 172-173.

Heikkilä, A.-M., 1999. Inherent Safety in Process Plant Design-An Index-Based Approach. VTT, Automation, Espoo, Finland (Ph.D. Thesis).

Hendershot, D.C., 2006. Lessons from human error incidents in process plant. Process Saf. Environ. Prot. 84, 174-178.

Hutton, M., 2017. Making the Link Between Process Safety and Sustainability, https://www.erm.com/en/insights/feature-articles/making-the-link-betweenprocess-safety-and-sustainability/. (Accessed 12 December 2017).

Jabbour, C.J.C., Jabbour, A.B.L.S., Sarkis, J., Godinho Filho, M., 2017. Unlocking the circular economy through new business models based on large-scale data: an integrative framework and research agenda. Technol. Forecast. Soc. Change, 1-7 (in press).

Jabbour, A.B.L.S., Jabbour, C.J.C., Foropon, C., Godinho Filho, M., 2018a. When titans meet -Can industry 4.0 revolutionise the environmentally sustainable manufacturing wave? The role of critical success factors. Technol. Forecast. Soc. Change, $1-8$ (in press).

Jabbour, A.B.L.S., Jabbour, C.J.C., Godinho Filho, M., Roubaud, D., 2018b. Industry 4.0 and the circular economy: a proposed research agenda and original roadmap for sustainable operations. Ann. Oper. Res., 1-16 (in press).

Jabbour, C.J.C., 2013. Environmental training in organizations: from a literature review to a framework for future research. Resour. Conserv. Recycl. 74, 144-155.

Johnson, D.L., Amborse, S.H., Bassett, T.J., Bowen, M.L., Crummey, D.E., Isaacson, J.S., Johnshon, D.N., Lamb, P., Saul, M., Winter-Nelson, A.E., 1997. Meanings of environmental terms. J. Environ. Qual. 26, 581-589.

Kagermann, H., Lukas, W., Wahlster, W., 2011. Industrie 4.0-Mit dem Internet der Dinger auf dem Weg zur 4. Industriellen Revolution. VDI Nachrichten, 13.

Kang, H.S., Barczack, G., Nicholas, J., Ledwith, A., Perks, H., 2012. An examination of new product development best practice. J. Prod. Innov. Manag. 29, 180-192.

Kehua, S., Li, J., Fu, H., 2011. Smart city and the applications. 2011 International Conference on Electronics Communications and Control (ICECC), 1028-1031.

Kesidou, E., Demirel, P., 2012. On the drivers of eco-innovations: empirical evidence from the UK. Res. Policy 41 (5), 862-870.

Khan, F., Rathnayaka, S., Ahmed, S., 2015. Methods and models in process safety and risk management Past, present and future. Process Saf. Environ. Prot. 98, $116-147$.

Kraft, M.E., 2017. Environmental Policy and Politics. Routledge, New York.

Lee, I., Lee, K., 2015. The Internet of Things (IoT) Applications, investments and challenges for enterprises. Bus. Horiz. 58, 431-440.

Lee, J., Kao, H., Yang, S., 2014. Service innovation and smart analytics for industry 4.0 and big data environment. Procedia CIRP 16, 3-8.

Lee, J., Bagheri, B., Kao, H.A., 2015. A cyber-physical systems architecture for industry 4.0-based manufacturing systems. Manufac. Lett. 3, 18-23.

Li, Z., Zongwei, M., Kuijp, T.J., Yuan, Z., Huang, L., 2014. A review of soil heavy metal pollution from mines in China: pollution and health risk assessment. Sci. Total Environ. 468-469, 843-853.

Lu, S.Q., Xie, G., Chen, Z., Han, X., 2015. The management of application of big data in Internet of Thing in Environmental Protection in China. IEEE First International Conference on Big Data Computing Service and Applications.

Luiz, J.V.R., Jugend, D., Jabbour, C.J.C., Luiz, O.R., De Souza, F.B., 2016. Ecodesign field of research throughout the world: mapping the territory by using an evolutionary lens. Scientometrics 109, 241-259.

Mazurkiewicz, P., 2004. Corporate environmental responsibility: is a common CSR framework possible? In: 24th Annual IAIA Conference, Vancouver, BC.

Mishra, S.A., Tijare, D.S., Asutkar, G.M., 2011. Design of energy aware air pollution monitoring system using WSN. Int. J. Adv. Eng. Tech 1 (2), 107-116.

Mittal, S., Khan, M.A., Romero, D., Wuest, T., 2017. Smart manufacturing: characteristics, technologies and enabling factors. Proc IMechE Part B: J Eng. Man., $1-20$.

Mongeon, P., Paul-Hus, A., 2016. The journal coverage of Web of Science and Scopus: a comparative analysis. Scientometrics 106, 213-228.

Nawahda, A, 2016. An assessment of adding value of traffic information and other attributes as part of its classifiers in a data mining tool set for predicting surface ozone levels. Process Saf. Envir. Prot. 99, 149-158.

OSHA, 2000. Process Safety Management. U.S. Department of Labor Occupational Safety and Health Administration, OSHA 31322000 (Reprinted). 
Peachey, B., 2008. Environmental stewardship -What does it mean? Process Saf. Environ. Prot. 86, 227-236.

Peters, H.P.F., Van Raan, A.F.J., 1993. Co-word-based science maps of chemical engineering: Part I: Representations by direct multidimensional scaling. Res. Policy $22(1), 23-45$.

Prause, G., 2015. Sustainable business models and structures for industry 4.0. J. Secur. Sustain. Issues 5 (2), 159-169.

Qi, R., Prem, K.P., Ng, D., Rana, M.A., Yun, G., Mannan, M.S., 2012. Challenges and needs for process safety in the new millennium. Process Saf. Environ. Prot. 90, 91-100.

Ramsauer, C., 2013. Industry 4.0. Die Produktion Der Zukunft. WINGbusiness 3/2003. pp. 6-12.

Shang, X., Liu, X., Xiong, G., Cheng, C., Ma, Y., Nyberg, T.R., 2013. Social manufacturing cloud service platform for the mass customization in apparel industry. IEEE International Conference on Service Operations and Logistics, and Informatics, 220-224.

Shibata, N., Kajikawa, Y., Takeda, Y., Matsushima, K., 2008. Detecting emerging research fronts based on topological measures in citation networks of scientific publications. Technovation 28 (11), 758-775.
Strandhagen, J.W., Alfnes, E., Strandhagen, J.O., Swahn, N., 2016. Importance of production environments when applying industry 4.0 to production logistics $-\mathrm{A}$ multiple case study. International Workshop of Advanced Manufacturing and Automation.

Su, K., Li, J., Fu, H., 2011. Smart city and the applications. IEEE International Conference on Electronics, Communications and Control (ICECC).

Taylor, J.R., 2017. Automated HAZOP revisited. Process Saf. Environ. Prot. 111, 635-651.

Van Eck, N.J., Waltman, L., 2014. Visualizing bibliometric networks. In: Ding, Y., Rousseau, R., Wolfram, D. (Eds.), Measuring Scholarly Impact: Methods and Practice. Springer, Berlin, pp. 285-320.

White, A., 2017. Environmental Protection and Management. Arcler Press LLC, Oakville.

Zhang, J., Knight, A., Duke, M., Northcott, K., Packer, M., Scales, P.J., Gray, S.R., 2016. A new integrated potable reuse process for a small remote community in Antarctica. Process Saf. Environ.Prot. 104, 196-208. 